\title{
Application of Bacillus thuringiensis as an effective tool for insect pest control
}

\author{
*Sajidmohammad A Saiyad \\ Biocontrol research laboratory, Anand Agricultural University, Anand
}

\section{Introduction}

Bacillus thuringiensis is a gram-positive spore-forming bacterium that produces crystalline proteins called delta-endotoxins during its stationary phase of growth. The crystal is released to the environment after lysis of the cell wall at the end of sporulation, and it can account for 20 to $30 \%$ of dry weight of the sporulated cells Fig. (1). This bacterium is distributed worldwide. The soil has been described as its main habitat; however it has also been isolated from foliage, water, storage grains, and dead insects, etc. Isolation of strains from dead insects has been the main source for commercially used varieties.

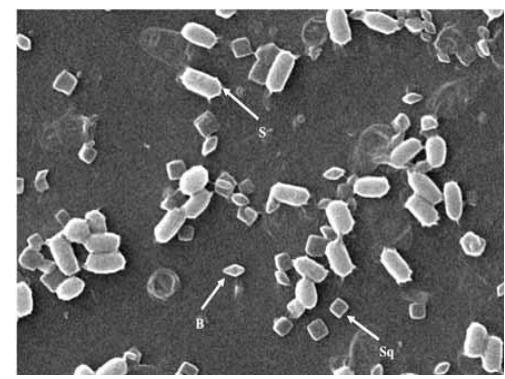

Fig. (1). Scanning electron micrograph of spores (S) and crystals of Bacillus thuringiensis strains

The leading biological insecticide is based on the common soil bacterium Bacillus thuringiensis (Bt). Formulations of these insecticides, containing mixtures of spores and crystals safely control insect pests of vegetables, Crops and forestry. Despite its high target specificity and environmentally favorable "green" characteristics, the Bt biopesticides market commands a small fraction of the global crop protection market. Features of Bt biopesticides limit their use in insect control. In contrast to contact insecticides, Bt biopesticides must be ingested by the target insect. The timing of Bt sprays is critical to attaining economic levels of insect control. Usually Bt is applied when early instar larvae are present, as older larvae are more tolerant. Bt sprays persist only a few days on the leaf surface. The chemistry of the leaf surface, proteinases and sunlight contribute to the degradation of Cry proteins. It is rare for a Bt insecticide to have greater efficacy than the best available chemical control. Hence, Bt adoption suffers at the hand of more efficacious chemical insecticides.

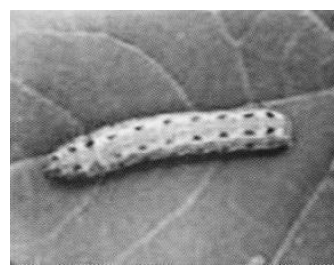

Healthy larvae

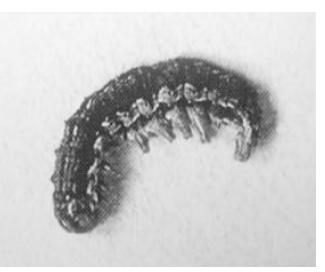

Larvae infected by

Bacillus

thuringiensis

Bt biopesticides have inherent advantages in certain pest control applications. They are used as a resistance management tools in insect control. Due to their distinct mode-of-action, they are alternated or combined with chemical pesticides. Bt is especially suited for specialty or 'high value crops.' The tightening of registration procedures for new chemical pesticides has led many of the larger crop protection companies to take the decision not to register products for use on specialty crops. Increased usage of Bt biopesticides will occur as organic markets expand and consumer demand for eco-friendly pest control alternatives in home gardens and treatments for high-value alternative crops. 


\section{Mode Of Action}

The Cry proteins comprise at least 50 subgroups with more than 200 members. The members belong to the larger group of Cry proteins is globular molecules with three structural domains connected by single linkers. The protoxins are characteristic of this family and have two different lengths. The $\mathrm{C}$-terminal extension found in the long protoxins is necessary for toxicity and is believed to play a role in the formation of the crystal within the bacterium.

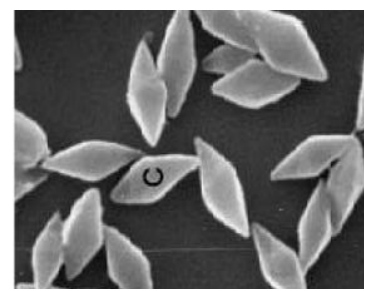

Crystal proteins

Their mode of action involves several events that must be completed several hours after ingestion in order to lead to insect death. Following ingestion, the crystals are solubilized by the alkaline conditions in the insect midgut and are subsequently proteolytically converted into a toxic core fragment. During proteolytic activation, peptides from the $\mathrm{N}$ terminus and $\mathrm{C}$ terminus are cleaved from the full protein. Activated toxin binds to receptors located on the apical microvillus membranes of epithelial midgut cells. For Cry1A toxins, at least four different binding sites have been described indifferent lepidopteran insects: a cadherin-like protein(CADR), a glycosylphosphatidyl-inositol(GPI)-anchoredaminopeptidase-N(APN), a GPI-anchored alkaline phosphatase (ALP) and a $270 \mathrm{kDa}$ glycoconjugate. After binding, toxin adopts a conformation allowing its insertion into the cell membrane. Subsequently, oligomerization occurs, and this oligomer forms a pore or ion channel induced by an increase in cationic permeability within the functional receptors contained on the brush borders membranes. This allows the free flux of ions and liquids, causing disruption of membrane transport and cell lysis, and leading to insect death. The complete nature of this process still remains unknown; however, it is believed that toxin aggregation occurs at the membrane surface after receptor binding, or alternatively only after the toxin inserts itself into the membrane. During the intoxication process, in lepidopterans as in coleopterans, many histopathological changes have been described, including swelling and disruption of the microvilli, vacuolization of the cytoplasm, hypertrophy of epithelial cells and necrosis of the nuclei.

\section{Bacillus thuringiensis based formulations for biopesticides}

Bacillus thuringiensis-based biopesticide production depends on high quality and high-efficiency formulation processes. Formulations must be safe and effective products, must be easy to use, and should have a long shelf life. The active ingredient in commercial formulations is the spore crystal complex, which is more effective to use and cheaper to obtain than the crystals alone, which are frequently used in experimental tests. The spore-crystal complex must be carried by suitable excipients that can function to protect the spore-crystal complex or to increase palatability to insects. In this sense, many studies reveal the inclusion of some kinds of components, related to enhance toxicity, or to enhance the attraction of insects. Many biodegradable compounds can be considered for use as inert carriers, depending on the type of formulation required.

The biopesticides must exhibit stability in storage, requiring improvement of its biological and physical properties. The use of additives is required in order to reduce evaporation and avoid formulation loss, and to provide a more extended coverage on and high adherence to foliage, improved dispersion, and a long residual effect. A great variety of ingredients have been employed to prepare formulations, including liquid or solid carriers, surfactants coadjuvants, fluidity agents, adherents, dispersants, stabilizers, moisturizers, attractants, and protective agents among others. An interesting and recent example of these kinds of inert ingredients is the superabsorbent starch graft co-polymer which combined with a B. thuringiensis strain among many other pesticides constitutes a novel formulation that could be applied in an agricultural environment. The biological activity of one particular biopesticide was enhanced when the antibiotic zwitter-mycin was added. This combination was also successful in pest control. Other compounds with such a synergism have also been used, in amounts effective for enhancing the pesticidal activity of a B. thuringiensis-based biopesticide. This can help reduce the amount of $B$. thuringiensis needed for a commercially effective biopesticides. Hobbs patented a pesticidal composition comprising a $B$. thuringiensis biopesticide present at ineffective levels, and a surfactant present at effective levels in order to control pests or to reduce the amount of the B. thuringiensis normally needed for commercially effective use. Bacillus thuringiensis-based products are classified according to their 
formulation. All products containing a blend of spores and crystals from a native strain are classified as firstgeneration products. These products constitute the majority of commercial products.

The second generation products are based on spores and crystals from a B. thuringiensis strain bearing artificially introduced genes coding for delta-endotoxins from several strains, in order to increase the activity spectrum against other insect pests. Formulations containing dead recombinant Pseudomonas fluorescens cells transformed with genes coding for delta-endotoxins are classified as third-generation products. An insecticide can be introduced into the market in different forms, depending on the insect to be controlled, the kind of crop, and the application method. In addition, the form of any formulation for release or dispensing into the environment is an important consideration. It depends on the pest to be controlled and the plant to which it will be applied. For other kinds of was a biopesticide formulation dispensed as a dry powder or tablet. Formulations contained B. thuringiensis var. israelensis and chemical dryers, dispersing agents, binding agents and moisturizing agents, protectors against sunlight; and optionally, diluents, lubricant and neutralizing agents. The biopesticide obtained was considered to be an ecologically safe product. As mentioned above, physical properties must always be considered when appropriate formulations are being developed. For successful field applications, properties such as fluidity and dispersion play an important role

Today, a great variety of $B$. thuringiensis-based biopesticides is commercially available for the control of a wide variety of agriculture and forestry pests. Many formulations already exist in the market; however, many patents relate to the use of different components that can further improve their insecticidal activity. In many cases, these products provide interesting advantages over previously developed insecticides.

\section{Reference}

[1] Ninfa M. Rosas-García :Biopesticide Production from Bacillus thuringiensis: An Environmentally Friendly Alternative ; Recent Patents on Biotechnology maxico2009, 3, 28-36

[2] Michael J. Adang; an overview of bacillus thuringiensis biopesticides

[3] Vincent Sanchis, Denis Bourguet; Bacillus thuringiensis: applications in agriculture and insect resistance management. A review; Agron. Sustain. Dev. 28 (2008) 11-20

[4] Roh, jongyul, jae young choi, ming shun li, byungraejin, and yeon ho je; Bacillus thuringiensis as a Specific, Safe, and Effective Tool for Insect Pest Control; J. Microbiol. Biotechnol. (2007), 17(4), 547-559

[5] AbidaBibi, Khalique Ahmed, NajmaAyub, SadiaAlam ;Production of low cost Bacillus thuringiensis basedbiopesticide for management of chickpea pod-borerHelicoverpaarmigera(Huebn) in Pakistan; Vol.5, No.11, 1139-1144 (2013)

[6] Fernando hercosvalicente, edmar de souzatuelher, mariaisabellasantos leite2, fernandalyonfreire and corinamacedovieira ; production of Bacillus thuringiensis biopesticide using commercial lab medium and agricultural by-products as nutrient sources.; RevistaBrasileira de Milho e Sorgo, v.9, n.1, p. 1-11, 2010

[7] RasoulMarzban; Investigation on the suitable isolate and medium for production of Bacillus thuringiensis; JBiopest. 5(2): 144-147

Sajidmohammad A Saiyad. "Application of Bacillus thuringiensis as an effective tool for insect pest control." IOSR Journal of Agriculture and Veterinary Science (IOSR-JAVS) 10.7 (2017): 27-29. 